\title{
TENETS OF AN EFFECTIVE AND EFFICIENT RESEARCH AND ANALYSIS PROGRAM FOR NASA
}

\author{
Community White Paper to the \\ Planetary Decadal Survey, 2021-2030
}

September 15, 2020

\author{
Authors \\ William F. Bottke \\ Harold F. Levison
}

\begin{tabular}{|c|c|}
\hline Endorser & Affiliation \\
\hline Joshua Kammer & Southwest Research Institute \\
\hline Stuart J. Robbins & Southwest Research Institute \\
\hline Steve Osterman & Southwest Research Institute \\
\hline Raluca Rufu & Southwest Research Institute \\
\hline Timothy R Holt & University of Southern Queensland \\
\hline Julien Salmon & Southwest Research Institute \\
\hline
\end{tabular}


In this white paper, we capture some of the major recommendations made by past National Academy reports regarding NASA's Research and Analysis (R\&A) program. We also make new recommendations that could be adopted in the upcoming Decadal Survey.

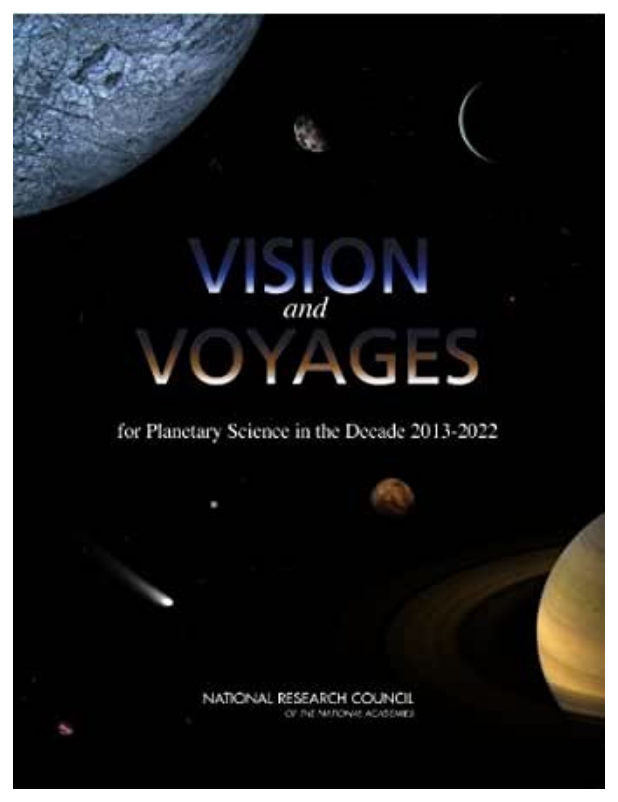

As discussed in the 2011 National Research Council planetary science decadal survey, Vision and Voyages for Planetary Science in the Decade 2013-2022 (Hereafter DS 2011), NASA's Research and Analysis Programs, managed by NASA’s Planetary Science Division, supports:

“... a broad range of planetary science activities, including the analysis of data from past and current spacecraft; laboratory research; theoretical, modeling, and computational studies; geological and astrobiological fieldwork in planetary analog environments on Earth; geological mapping of planetary bodies; analysis of data from Earth- and space-based telescopes; and development of flight instruments and technology needed for future planetary science missions."

They are of critical importance to the health and welfare of an effective NASA portfolio of science and exploration. As stated in DS 2011:

"The research related to planetary missions begins well before a mission is formulated and funded, and continues long after it is over. Research provides the foundation for interpreting data collected by spacecraft, as well as the guidance and context for identifying new scientifically compelling missions. Ground-based observations can identify new targets for 
future missions, and experimental and theoretical results can pose new questions for these missions to answer. Research and analysis programs also allow the maximum possible science return to be harvested from missions. And along with analysis of spacecraft data, the portfolios of research and analysis programs include laboratory experiments, theoretical studies, fieldwork using Earth analogs, planetary geologic mapping, and analysis of data from Earth-based telescopes. All of these efforts are crucially important to NASA's long-term science goals, and all require funding.

One example of the success of these programs can be seen in the leadership of NASA missions. For example, the PIs of both the Lucy and Psyche missions were previously funded by NASA's R\&A programs. They leveraged the knowledge and understanding of planetary science that can only be obtained through an active basic research program to develop mission concepts that will deliver the most impactful science from the limited NASA mission funds.

In addition to leading to the development of exciting mission concepts, the activities funded by R\&A often include students and young scientists who are directly involved in the research. A major goal of R\&A programs is thus to help train future generations of space scientists and engineers.

A good share of planetary research is paid for through small grants. In order to stay funded, many researchers must spend considerable time writing proposals instead of doing science. This places a premium on keeping NASA's R\&A program healthy enough that researchers can stay productive -- spending their time doing research rather than writing proposals. In fact, this goal was documented in the funding priority list laid out in DS 2011:

"If cuts to [NASA] programs are necessary, the first approach should be descoping or delaying large strategic (flagship) missions. Changes to the New Frontiers or Discovery programs should be considered only if adjustments to large strategic missions cannot solve the problem. And high priority should be placed on preserving funding for research and analysis programs and for technology development." 


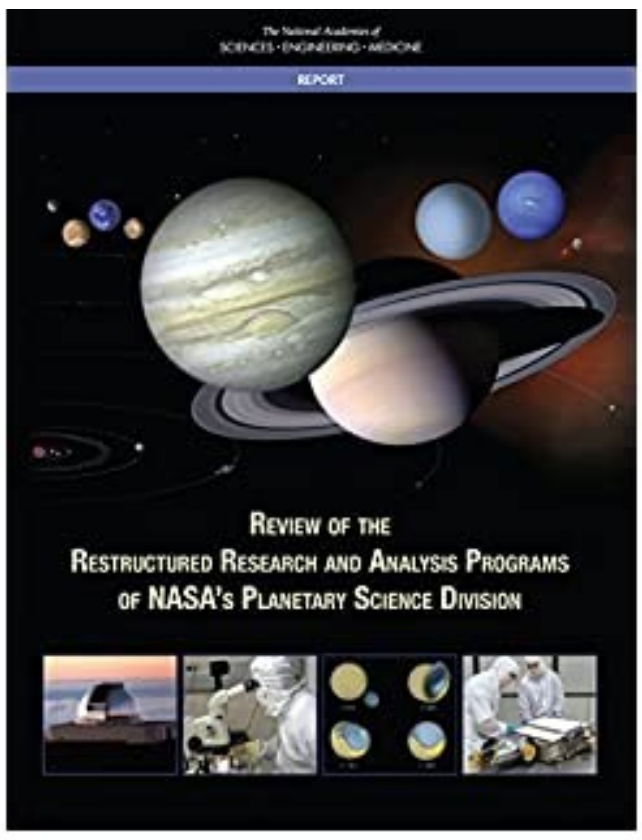

A second National Academies report in 2017, "Review of the Restructured Research and Analysis Programs of NASA's Planetary Science Division (hereafter Review 2017), discussed aspects of the R\&A proposal-review process and made recommendations that they felt NASA should adopt. We repeat them here.

"The committee is strongly of the opinion that its concerns provide important input to NASA on how to improve the existing program...

... With respect to the procedures followed by PSD in the implementation of the current program, the committee recommends the following:

Recommendation: In conducting scientific peer reviews of research proposals, NASA's Planetary Science Division should engage the services of several (at least two or three) external (mail) reviewers well in advance of panel reviews. These reviews are critical to a fair and effective proposal evaluation process, particularly when the review panels have a more interdisciplinary character. The panel chair and group chiefs, if recruited early, can take the lead in identification of appropriate external reviewers."

This recommendation was based on the fact that subpanels often do not have the appropriate expertise and need to take advantage of external reviewers to fill the expertise gap. It is often observed on subpanels that only an external referee will understand what approaches will or will not work -- or whether a given project has or has not been done before. Ultimately, external reviewers are critical to the review process because they allow panels to tap into specialized expertise. 
"Recommendation: NASA's Planetary Science Division should expeditiously complete establishment of the process for reconsideration of proposal selection decisions, develop and implement a formal mechanism to track debriefing and reconsideration requests across program elements, and inform the community about the process. More transparency in this area can provide the planetary science community with greater confidence that NASA has appropriate checks and balances in the selection process."

While we acknowledge that proposal review debriefs can be obtained from NASA program managers, we argue that the process is still opaque to most scientists. We would like to see presentations at major planetary science conferences on the next steps that should be followed by those with failed proposals. 


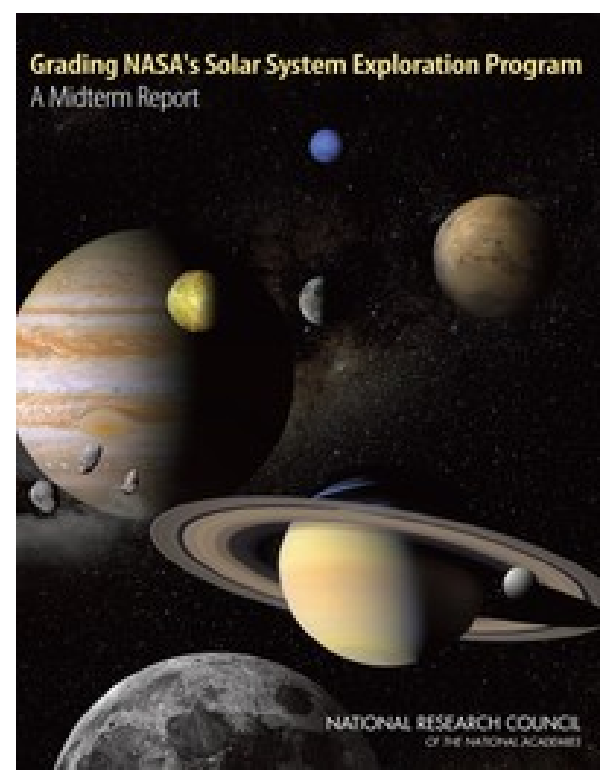

A third National Academy report, "Visions into Voyages for Planetary Science in the Decade 2013-2022: A Midterm Review (2018)", made two recommendations for R\&A that have yet to be implemented:

"Recommendation: The next decadal survey committee should work with NASA to better understand the categorization and tracking of the budget for each of the research and analysis program elements, specifically providing insight into the budget for (1) principal investigator (PI)-led, competed, basic research and data analysis; (2) ground-based observations; (3) infrastructure and management; and (4) institutional or field center support. Also, the next decadal survey should be unambiguous when stipulating programs and recommended levels of spending."

"Recommendation: A formal assessment by NASA of how well the program structure and funding of the virtual institutes are aligned with the Planetary Science Division's science goals should be conducted on a regular basis, appropriately phased to the cycle of decadal surveys and midterm reviews." 


\section{New Recommendations for the Upcoming Decadal Survey}

Finally, the authors of this white paper wish to make several additional recommendations that can help NASA's R\&A programs achieve their goals. To make them easier to follow, we present them in an itemized format:

- NASA’s planetary research and analysis programs are heavily oversubscribed.

o Major programs, such as Solar System Workings, had a very low selection rate in FY20 (11\%) because of various NASA budget issues. NASA program managers have also made recent predictions that these same programs will have similarly low selection rates in FY21 and possibly FY22.

o The existing funding crisis in R\&A is dangerous for NASA if it is to meet its ambitious science and exploration goals. It threatens to drive out of the field: (i) the next generation of young scientists and (ii) older scientists with critical expertise already paid for by NASA. One consequence may be that existing and new missions are less likely to achieve top Decadal Science goals. Another may be that NASA takes much longer to meet these goals, with the net cost to the program being higher overall.

o We recommend that NASA ensures that high quality proposals, namely those achieving grades of Excellent, Excellent/Very Good, or Very Good, will be largely funded. To do this, we argue that an appropriate selection rate for major R\&A programs distributing small grants should be at least 20\%. The programs should also leave room for risky high impact proposals to be funded within existing programs.

- The soft money community plays a critical, unacknowledged role in meeting the Decadal Survey's goals.

o Soft money scientists are valuable because they have fewer institutional obligations and so therefore can spend nearly all of their time on research. We argue there is value in allowing some fraction of the research community to focus their time on high impact problems without distractions.

o Soft money science attracts those that are risk takers by design. They are willing to take the prospect of not having secure funds in order to spend more time doing research. They arguably provide NASA with a more flexible community for doing research than hard money scientists because, by necessity, they are more likely to propose to new programs where NASA has set out its latest objectives.

o Many changes to the R\&A programs that NASA has recently put into place put the soft money community at greater risk. We recommend that these changes be reevaluated with this consequence in mind. 
- If NASA is serious about creating a more diverse workforce - one that looks more like the US workforce - it should consider augmenting opportunities for soft money scientists.

o Soft money scientists accommodate a wider diversity of lifestyles and life conditions (those that need to stay home to take care of children or the elderly; those that need to follow their spouse to a rural location far from a college, university or NASA center). Allowing for such flexibility is critical for increasing underrepresented groups within the field.

o The soft money community is easier to enter compared to the always limited number of faculty and civil servant positions. From soft money positions, underrepresented groups can focus on research and gain valuable experience that may eventually translate into them becoming future mission/instrument PIs, taking on NASA leadership positions, and so on.

- We recommend that R\&A proposals be required to make an explicit case that they are addressing the science priorities of the Decadal Survey.

o This could be a major evaluation consideration for individual small grant proposals. We argue this addition would guarantee that funded proposals will have a higher impact toward Decadal Survey goals.

- We argue that several tenets of a healthy R\&A program should have the following attributes.

o Given the consolidation of many different R\&A programs in the past, PIs must be allowed to have multiple grants within the same program. While NASA programs currently allow this to occur, it is not a rule, and some are against the practice.

o Given the new push for no due dates on proposals, NASA must guarantee that all proposals will be reviewed within 3 months of submission. This will ensure timely research topics are addressed in an expedient manner.

o NASA should once again consider 4-year and 5-year grants. Three years is too short for certain kinds of science to be achieved.

o Individual R\&A programs should not be evaluated on how many PIs are funded. This puts pressure on the program manager to fund proposals with smaller budgets, and is a bias against older, more experienced researchers. NASA should instead develop some alternative metric to evaluate success of a program, one that considers scientific productivity and scientific impact to the Decadal Survey goals.

o Getting good review panel members is difficult because of heightened conflict of interest rules. Large programs, such as Solar System Workings, naturally have more conflicts because more people apply to these programs. As the situation now sits, many qualified reviewers cannot participate in the review process. A solution must be found to allow more qualified panel members to participate than the current rules allow. 